\title{
Research Article \\ Sufficiency and Duality for Multiobjective Programming under New Invexity
}

\author{
Yingchun Zheng and Xiaoyan Gao \\ College of Science, Xian University of Science and Technology, Xian 710054, China \\ Correspondence should be addressed to Yingchun Zheng; zhychun1979@163.com
}

Received 24 March 2016; Accepted 28 August 2016

Academic Editor: Yakov Strelniker

Copyright (c) 2016 Y. Zheng and X. Gao. This is an open access article distributed under the Creative Commons Attribution License, which permits unrestricted use, distribution, and reproduction in any medium, provided the original work is properly cited.

\begin{abstract}
A class of multiobjective programming problems including inequality constraints is considered. To this aim, some new concepts of generalized $(F, P)$-type I and $(F, P)$-type II functions are introduced in the differentiable assumption by using the sublinear function $F$. These new functions are used to establish and prove the sufficient optimality conditions for weak efficiency or efficiency of the multiobjective programming problems. Moreover, two kinds of dual models are formulated. The weak dual, strong dual, and strict converse dual results are obtained under the aforesaid functions.
\end{abstract}

\section{Introduction}

The field of multiobjective programming, also called vector programming, has grown remarkably in different directions since the 1980s. Many researchers have been interested in the optimality conditions and duality results for the weak efficient solution and efficient solution of the multiobjective programming problems. A large literature was developed around the sufficiency and duality in multiobjective optimization [1]. In [2], Jayswal obtained the Kuhn-Tucker type sufficient optimality conditions for a feasible solution to be an efficient solution and the Mond-Weir type duality results are also presented. More specifically, Gao [3] considered the nonsmooth multiobjective semi-infinite programming and obtained several sufficient conditions and duality results. Also, Bae et al. [4] established duality theorems for nondifferentiable multiobjective programming problems under generalized convexity assumptions. Recently, Kim and Lee [5] introduced the nonsmooth multiobjective programming problems involving locally Lipschitz functions and support functions. For more descriptions of the multiobjective programming, we refer to [6-9].

Furthermore, various types of generalizations of convexity theory have played an important role in the evolution of the multiobjective programming. During the past decades, the generalizations of invexity were enriched with and without differentiability assumptions. For example, we can see [10-12]. In particular, Nahak and Mohapatra [13] introduced the concept of $\rho-(\eta, \theta)$-invexity function and discussed a class of multiobjective programming problems by using the new generalized functions. Padhan and Nahak [14] introduced higher-order $\rho$ - $(\eta, \theta)$-invexity functions for studying two different pairs of higher-order symmetric dual programs. In [15], Antczak extended the concept of $(\phi, \rho)$ invexity for differentiable optimization problems to the case of mathematical programming problems with locally Lipschitz functions. In [16], Antczak and Stasiak introduced the concept of $(\phi, \rho)$-invexity for strong compact Lipschitz mappings in Banach spaces. Sufficient optimality conditions and Mond-Weir duality theorems are derived by the assumption of generalized nonsmooth $(\phi, \rho)$-invexity between Banach spaces. In [17], based upon the $F$-convexity and $\rho$-convexity, the authors defined the $(\phi, \rho)$-V-type I functions to consider a class of nonsmooth multiobjective programming problems. The invexity of functions is more useful in the research of optimization.

In this paper, we consider the multiobjective programming problems. The new class of generalized invexity functions, namely, pseudoinvex $(F, P)$-type I (pseudoinvex $(F, P)$ type II, etc.) are introduced. The sufficient optimality conditions are obtained. Then weak, strong, and strict converse dual results are also established for two types of dual models 
related to multiobjective programming problems involving the new generalized invex functions.

\section{Notations and Preliminaries}

Throughout the paper, we use the following conventions for vectors in $R^{n}$ :

$$
\begin{aligned}
& x \leqq y \quad \text { if and only } x_{i} \leq y_{i}, \forall i=1,2, \ldots, n ; \\
& x \leq y \quad \text { if and only } x_{i} \leqq y_{i}, \forall i=1,2, \ldots, n, x \neq y ; \\
& x<y \quad \text { if and only } x_{i}<y_{i}, \forall i=1,2, \ldots, n .
\end{aligned}
$$

In this paper, we consider the following multiobjective programming problem:

$$
\begin{array}{ll}
\text { Minimize } & f(x)=\left(f_{1}(x), f_{2}(x), \ldots, f_{k}(x)\right) \\
\text { subject to } & g_{j}(x) \leqq 0, \quad j=1,2, \ldots, m \\
& x \in X
\end{array}
$$

where $X \subseteq R^{n}$ is an open set and $f_{i}: X \rightarrow R, i \in K=$ $\{1,2, \ldots, k\}$ and $g_{j}: X \rightarrow R, j \in M=\{1,2, \ldots, m\}$ are differentiable on $X$. Let $X_{0}=\left\{x \mid g_{j}(x) \leqq 0, j \in M\right\}$ be the set of all feasible solutions of (MP).
Definition 1. A feasible solution $\bar{x} \in X_{0}$ of (MP) is said to be a weakly efficient solution for (MP), if there exists no other $x \in X_{0}$, such that

$$
f(x)<f(\bar{x}) .
$$

Definition 2. A feasible solution $\bar{x} \in X_{0}$ of (MP) is said to be an efficient solution for (MP), if there exists no other $x \in X_{0}$, such that

$$
f(x) \leq f(\bar{x}) .
$$

Definition 3. A function $F: X \times X \times R^{n} \rightarrow R$ is sublinear if, for any $x, \bar{x} \in X$,

$$
\begin{aligned}
F\left(x, \bar{x} ; a_{1}+a_{2}\right) \leqq F\left(x, \bar{x} ; a_{1}\right)+F\left(x, \bar{x} ; a_{2}\right), & \\
& \forall a_{1}, a_{2} \in R^{n}, \\
F(x, \bar{x} ; l a)=l F(x, \bar{x} ; a), &
\end{aligned}
$$

$$
\forall l \in R, l \geqq 0, a \in R^{n} .
$$

Remark 4. It should be noted that $F(x, \bar{x} ; 0)=0$.

Let $f$ and $g$ be differentiable at a given point $\bar{x} \in X, \eta$ : $X \times X \rightarrow R^{n}, \alpha_{i}, \beta_{j}: X \times X \rightarrow R_{+} \backslash\{0\}, d: X \times X \rightarrow$ $R, \rho_{i}, \tau_{j} \in R, b: X \times X \rightarrow R_{+} \backslash\{0\}, P \in R^{n}, i \in K, j \in M$.

Definition 5. $(f, g)$ is said to be pseudoinvex $(F, P)$-type I at $\bar{x} \in X$, if there exists functions $b, \alpha_{i}, \beta_{j}, \eta, d$ and $\rho_{i}, \tau_{j} \in R, P \in$ $R^{n}$, such that each $x \in X$; the following inequalities hold:

$$
b(x, \bar{x})(f(x)-f(\bar{x}))<0 \Longrightarrow\left\{\begin{array}{l}
F\left(x, \bar{x} ; \alpha_{i}(x, \bar{x}) \eta^{T}(x, \bar{x}) \nabla f_{i}(\bar{x}) P\right)+\rho_{i} d^{2}(x, \bar{x})<0, \quad i \in K, \\
F\left(x, \bar{x} ; \beta_{j}(x, \bar{x}) \eta^{T}(x, \bar{x}) \nabla g_{j}(\bar{x}) P\right)+\tau_{j} d^{2}(x, \bar{x})<0, \quad j \in M
\end{array}\right.
$$

Example 6. Let $X=[-1,1] \subset R$. Let the functions $f: X \rightarrow$ $R, g: X \rightarrow R$, and $b: X \times X \rightarrow R_{+} \backslash\{0\}$ be defined by

$$
\begin{gathered}
f(x)=e^{x}-3 x, \\
g(x)=-x^{3}-2 x-5, \\
b(x, \bar{x})=x^{2}+\bar{x}^{2}+1,
\end{gathered}
$$

and the functions $F: X \times X \times R \rightarrow R, \eta: X \times X \rightarrow R$ be given by

$$
\begin{gathered}
F(x, \bar{x} ; a)=a\left(2-\bar{x}^{2}\right), \\
\eta(x, \bar{x})=2 x-\bar{x} .
\end{gathered}
$$

Moreover, the functions $\alpha: X \times X \rightarrow R_{+} \backslash\{0\}, \beta: X \times X \rightarrow$ $R_{+} \backslash\{0\}$, and $d: X \times X \rightarrow R$ are defined by

$$
\begin{aligned}
& \alpha(x, \bar{x})=\bar{x}^{2}+1, \\
& \beta(x, \bar{x})=\bar{x}+2, \\
& d(x, \bar{x})=x-\bar{x} .
\end{aligned}
$$

Then, let

$$
\begin{aligned}
& \rho=-1, \\
& \tau=-2, \\
& P=1 .
\end{aligned}
$$

Now, we have

$$
b(x, \bar{x})(f(x)-f(\bar{x}))<0, \quad \text { where } \bar{x}<x .
$$

Then

$$
\begin{aligned}
& F\left(x, \bar{x} ; \alpha(x, \bar{x}) \eta^{T}(x, \bar{x}) \nabla f(\bar{x}) P\right)+\rho d^{2}(x, \bar{x})<0, \\
& F\left(x, \bar{x} ; \beta(x, \bar{x}) \eta^{T}(x, \bar{x}) \nabla g(\bar{x}) P\right)+\tau d^{2}(x, \bar{x})<0 .
\end{aligned}
$$

Hence $(f, g)$ is pseudoinvex $(F, P)$-type I at $u \in X$, where $u<x, x \in X$.

Definition 7. $(f, g)$ is said to be pseudoinvex $(F, P)$-type II at $\bar{x} \in X$, if there exists functions $b, \alpha_{i}, \beta_{j}, \eta, d$ and $\rho_{i}, \tau_{j} \in$ $R, P \in R^{n}$, such that each $x \in X$; the following inequalities hold: 


$$
b(x, \bar{x})(f(x)-f(\bar{x})) \leq 0 \Longrightarrow \begin{cases}F\left(x, \bar{x} ; \alpha_{i}(x, \bar{x}) \eta^{T}(x, \bar{x}) \nabla f_{i}(\bar{x}) P\right)+\rho_{i} d^{2}(x, \bar{x})<0, \quad i \in K, \\ F\left(x, \bar{x} ; \beta_{j}(x, \bar{x}) \eta^{T}(x, \bar{x}) \nabla g_{j}(\bar{x}) P\right)+\tau_{j} d^{2}(x, \bar{x})<0, \quad j \in M\end{cases}
$$

Definition 8. $(f, g)$ is said to be pseudoquasi-invex $(F, P)$ type I at $\bar{x} \in X$, if there exists functions $b, \alpha_{i}, \beta_{j}, \eta, d$ and $\rho_{i}, \tau_{j} \in R, P \in R^{n}$, such that each $x \in X$; the following inequalities hold:

$$
b(x, \bar{x})(f(x)-f(\bar{x}))<0 \Longrightarrow\left\{\begin{array}{l}
F\left(x, \bar{x} ; \alpha_{i}(x, \bar{x}) \eta^{T}(x, \bar{x}) \nabla f_{i}(\bar{x}) P\right)+\rho_{i} d^{2}(x, \bar{x})<0, \quad i \in K \\
F\left(x, \bar{x} ; \beta_{j}(x, \bar{x}) \eta^{T}(x, \bar{x}) \nabla g_{j}(\bar{x}) P\right)+\tau_{j} d^{2}(x, \bar{x}) \leqq 0, \quad j \in M
\end{array}\right.
$$

Example 9. Let $f: R^{2} \rightarrow R^{2}, g: R^{2} \rightarrow R$, and $b: R^{2} \times R^{2} \rightarrow$ $R_{+} \backslash\{0\}$ be defined by

$$
\begin{aligned}
f_{1}(x) & =x_{1} e^{2 x_{2}}, \\
f_{2}(x) & =x_{2}^{3}\left(x_{1}^{2}+1\right), \\
g(x) & =2 x_{1}+x_{2}^{2}-3, \\
b(x, \bar{x}) & =x_{1}^{2}+x_{1}+1,
\end{aligned}
$$

and the functions $F: R^{2} \times R^{2} \times R^{2} \rightarrow R$ and $\eta: R^{2} \times R^{2} \rightarrow R^{2}$ be given by

$$
\begin{aligned}
& F(x, \bar{x} ; a)=a^{T} c(x, \bar{x}) \\
& \text { where } c(x, \bar{x})=\left(x_{1}-x_{2}, x_{2}\right)^{T} \text { is a vector function. } \\
& \quad \eta(x, \bar{x})=\left(x_{1}^{2}+x_{2}^{2}, x_{1}-2 x_{2}+1\right)^{T} .
\end{aligned}
$$

Moreover, the functions $\alpha_{i}: R^{2} \times R^{2} \rightarrow R_{+} \backslash\{0\}, \beta: R^{2} \times R^{2} \rightarrow$ $R_{+} \backslash\{0\}$, and $d: R^{2} \times R^{2} \rightarrow R$ are defined by

$$
\begin{aligned}
& \alpha_{1}(x, \bar{x})=\alpha_{2}(x, \bar{x})=x_{1}^{2}+e^{\cos x_{2}}, \\
& \beta(x, \bar{x})=1, \\
& d(x, \bar{x})=x_{1}+x_{2} .
\end{aligned}
$$

Then, let

$$
\begin{aligned}
\rho_{1} & =\rho_{2}=\tau=-1, \\
P & =(1,1)^{T} .
\end{aligned}
$$

It is easy to see that $(f, g)$ is pseudoquasi-invex $(F, P)$-type I at the point $\bar{x}=(0,0)$ with respect to $b, \alpha_{i}(i=1,2), \beta, \eta, \rho_{i}(i=$ $1,2), \tau, P$, and $d$.

Definition 10. $(f, g)$ is said to be pseudoquasi-invex $(F, P)$ type II at $\bar{x} \in X$, if there exists functions $b, \alpha_{i}, \beta_{j}, \eta, d$, and $\rho_{i}, \tau_{j} \in R, P \in R^{n}$, such that each $x \in X$; the following inequalities hold:

$$
b(x, \bar{x})(f(x)-f(\bar{x})) \leq 0 \Longrightarrow\left\{\begin{array}{l}
F\left(x, \bar{x} ; \alpha_{i}(x, \bar{x}) \eta^{T}(x, \bar{x}) \nabla f_{i}(\bar{x}) P\right)+\rho_{i} d^{2}(x, \bar{x})<0, \quad i \in K, \\
F\left(x, \bar{x} ; \beta_{j}(x, \bar{x}) \eta^{T}(x, \bar{x}) \nabla g_{j}(\bar{x}) P\right)+\tau_{j} d^{2}(x, \bar{x}) \leqq 0, \quad j \in M
\end{array}\right.
$$

Definition 11. $(f, g)$ is said to be quasipseudo-invex $(F, P)$ type I at $\bar{x} \in X$, if there exists functions $b, \alpha_{i}, \beta_{j}, \eta, d$, and $\rho_{i}, \tau_{j} \in R, P \in R^{n}$, such that each $x \in X$; the following inequalities hold:

$$
b(x, \bar{x})(f(x)-f(\bar{x}))<0 \Longrightarrow \begin{cases}F\left(x, \bar{x} ; \alpha_{i}(x, \bar{x}) \eta^{T}(x, \bar{x}) \nabla f_{i}(\bar{x}) P\right)+\rho_{i} d^{2}(x, \bar{x}) \leqq 0, & i \in K, \\ F\left(x, \bar{x} ; \beta_{j}(x, \bar{x}) \eta^{T}(x, \bar{x}) \nabla g_{j}(\bar{x}) P\right)+\tau_{j} d^{2}(x, \bar{x})<0, & j \in M\end{cases}
$$

Definition 12. $(f, g)$ is said to be quasipseudo-invex $(F, P)$ type II at $\bar{x} \in X$, if there exists functions $b, \alpha_{i}, \beta_{j}, \eta, d$, and $\rho_{i}, \tau_{j} \in R, P \in R^{n}$, such that each $x \in X$; the following inequalities hold:

$$
b(x, \bar{x})(f(x)-f(\bar{x})) \leq 0 \Longrightarrow \begin{cases}F\left(x, \bar{x} ; \alpha_{i}(x, \bar{x}) \eta^{T}(x, \bar{x}) \nabla f_{i}(\bar{x}) P\right)+\rho_{i} d^{2}(x, \bar{x}) \leqq 0, & i \in K, \\ F\left(x, \bar{x} ; \beta_{j}(x, \bar{x}) \eta^{T}(x, \bar{x}) \nabla g_{j}(\bar{x}) P\right)+\tau_{j} d^{2}(x, \bar{x})<0, & j \in M\end{cases}
$$




\section{Sufficient Optimality Conditions}

Now, we establish sufficient optimality conditions for the considered optimization problem (MP) under the new invexity.

Theorem 13. Let $\bar{x} \in R^{n}$ be a feasible solution in problem (MP). Suppose that

(i) there exists $\lambda_{i} \geqq 0, \sum_{i=1}^{k} \lambda_{i}=1, \mu_{j} \geqq 0, i \in K, j \in M$, such that

$$
\sum_{i=1}^{k} \lambda_{i} \nabla f_{i}(\bar{x})+\sum_{j=1}^{m} \mu_{j} \nabla g_{j}(\bar{x})=0 ;
$$

(ii) $(f, g)$ is pseudoinvex $(F, P)$-type I at $\bar{x}$;

(iii) $\sum_{i=1}^{k}\left(\lambda_{i} \rho_{i} / \alpha_{i}(x, \bar{x})\right)+\sum_{j=1}^{m}\left(\mu_{j} \tau_{j} / \beta_{j}(x, \bar{x})\right) \geqq 0$.

Then $\bar{x}$ is a weakly efficient solution for (MP).

Proof. Suppose contrary to the result that $\bar{x}$ is not a weakly efficient solution to (MP). Then there exists $x \in X_{0}$ such that

$$
f(x)<f(\bar{x}) .
$$

With $b(x, \bar{x})>0$, the above inequality yields

$$
b(x, \bar{x})(f(x)-f(\bar{x}))<0,
$$

from hypothesis (ii), which implies

$$
\begin{aligned}
& F\left(x, \bar{x} ; \alpha_{i}(x, \bar{x}) \eta^{T}(x, \bar{x}) \nabla f_{i}(\bar{x}) P\right)+\rho_{i} d^{2}(x, \bar{x})<0, \\
& i \in K, \\
& F\left(x, \bar{x} ; \beta_{j}(x, \bar{x}) \eta^{T}(x, \bar{x}) \nabla g_{j}(\bar{x}) P\right)+\tau_{j} d^{2}(x, \bar{x}) \\
& \quad<0, \quad j \in M .
\end{aligned}
$$

By sublinearity of $F$ with $\alpha_{i}(x, \bar{x})>0$ and $\beta_{j}(x, \bar{x})>0$, inequalities (24) yield

$$
\begin{aligned}
& F\left(x, \bar{x} ; \eta^{T}(x, \bar{x}) \nabla f_{i}(\bar{x}) P\right)<-\frac{\rho_{i}}{\alpha_{i}(x, \bar{x})} d^{2}(x, \bar{x}), \\
& i \in K \text {, } \\
& F\left(x, \bar{x} ; \eta^{T}(x, \bar{x}) \nabla g_{j}(\bar{x}) P\right)<-\frac{\tau_{j}}{\beta_{j}(x, \bar{x})} d^{2}(x, \bar{x}), \\
& j \in M \text {. }
\end{aligned}
$$

Using $\lambda_{i} \geqq 0, \sum_{i=1}^{k} \lambda_{i}=1$, and $\mu_{j} \geqq 0, j \in M$, along with the sublinearity of $F$, from inequality (25), we get

$$
\begin{gathered}
F\left(x, \bar{x} ; \eta^{T}(x, \bar{x})\left(\sum_{i=1}^{k} \lambda_{i} \nabla f_{i}(\bar{x})\right) P\right) \\
<-\sum_{i=1}^{k} \frac{\lambda_{i} \rho_{i}}{\alpha_{i}(x, \bar{x})} d^{2}(x, \bar{x}),
\end{gathered}
$$

$$
\begin{aligned}
& F\left(x, \bar{x} ; \eta^{T}(x, \bar{x})\left(\sum_{j=1}^{m} \mu_{j} \nabla g_{j}(\bar{x})\right) P\right) \\
& \leqq-\sum_{j=1}^{m} \frac{\mu_{j} \tau_{j}}{\beta_{j}(x, \bar{x})} d^{2}(x, \bar{x}) .
\end{aligned}
$$

By the sublinearity of $F$, we sum (26) to obtain

$$
\begin{gathered}
F\left(x, \bar{x} ; \eta^{T}(x, \bar{x})\left(\sum_{i=1}^{k} \lambda_{i} \nabla f_{i}(\bar{x})+\sum_{j=1}^{m} \mu_{j} \nabla g_{j}(\bar{x})\right) P\right) \\
<-\left(\sum_{i=1}^{k} \frac{\lambda_{i} \rho_{i}}{\alpha_{i}(x, \bar{x})}+\sum_{j=1}^{m} \frac{\mu_{j} \tau_{j}}{\beta_{j}(x, \bar{x})}\right) d^{2}(x, \bar{x}),
\end{gathered}
$$

from hypothesis (iii), which follows

$$
\begin{aligned}
& F\left(x, \bar{x} ; \eta^{T}(x, \bar{x})\left(\sum_{i=1}^{k} \lambda_{i} \nabla f_{i}(\bar{x})+\sum_{j=1}^{m} \mu_{j} \nabla g_{j}(\bar{x})\right) P\right) \\
& \quad<0 .
\end{aligned}
$$

On the other hand, the hypothesis (i) implies

$$
\begin{aligned}
& F\left(x, \bar{x} ; \eta^{T}(x, \bar{x})\left(\sum_{i=1}^{k} \lambda_{i} \nabla f_{i}(\bar{x})+\sum_{j=1}^{m} \mu_{j} \nabla g_{j}(\bar{x})\right) P\right) \\
& \quad=0
\end{aligned}
$$

which contradicts (28). Hence the conclusion of the theorem is established.

Theorem 14. Let $\bar{x} \in R^{n}$ be a feasible solution in problem (MP). Suppose that

(i) there exists $\lambda_{i} \geqq 0, \sum_{i=1}^{k} \lambda_{i}=1, \mu_{j} \geqq 0, i \in K, j \in M$, such that

$$
\sum_{i=1}^{k} \lambda_{i} \nabla f_{i}(\bar{x})+\sum_{j=1}^{m} \mu_{j} \nabla g_{j}(\bar{x})=0
$$

(ii) $(f, g)$ is pseudoinvex $(F, P)$-type II at $\bar{x}$;

(iii) $\sum_{i=1}^{k}\left(\lambda_{i} \rho_{i} / \alpha_{i}(x, \bar{x})\right)+\sum_{j=1}^{m}\left(\mu_{j} \tau_{j} / \beta_{j}(x, \bar{x})\right) \geqq 0$.

Then $\bar{x}$ is an efficient solution for (MP).

Proof. By the way of contradiction, suppose that $\bar{x}$ is not an efficient solution for (MP). Then there exists $x \in X_{0}$ such that

$$
f(x) \leq f(\bar{x}) .
$$

With $b(x, \bar{x})>0$, the above inequality yields

$$
b(x, \bar{x})(f(x)-f(\bar{x})) \leq 0,
$$


by hypothesis (ii), which follows

$$
\begin{aligned}
& F\left(x, \bar{x} ; \alpha_{i}(x, \bar{x}) \eta^{T}(x, \bar{x}) \nabla f_{i}(\bar{x}) P\right)+\rho_{i} d^{2}(x, \bar{x})<0, \\
& i \in K, \\
& F\left(x, \bar{x} ; \beta_{j}(x, \bar{x}) \eta^{T}(x, \bar{x}) \nabla g_{j}(\bar{x}) P\right)+\tau_{j} d^{2}(x, \bar{x}) \\
& \quad<0, \quad j \in M .
\end{aligned}
$$

Using $\alpha_{i}(x, \bar{x})>0, \beta_{j}(x, \bar{x})>0$ and $\lambda_{i} \geqq 0, \sum_{i=1}^{k} \lambda_{i}=1$, $\mu_{j} \geqq 0, j \in M$, along with the sublinearity of $F$, inequality (33) yields

$$
\begin{aligned}
& F\left(x, \bar{x} ; \eta^{T}(x, \bar{x})\left(\sum_{i=1}^{k} \lambda_{i} \nabla f_{i}(\bar{x})\right) P\right) \\
& <-\sum_{i=1}^{k} \frac{\lambda_{i} \rho_{i}}{\alpha_{i}(x, \bar{x})} d^{2}(x, \bar{x}), \\
& F\left(x, \bar{x} ; \eta^{T}(x, \bar{x})\left(\sum_{j=1}^{m} \mu_{j} \nabla g_{j}(\bar{x})\right) P\right) \\
& \leqq-\sum_{j=1}^{m} \frac{\mu_{j} \tau_{j}}{\beta_{j}(x, \bar{x})} d^{2}(x, \bar{x}) .
\end{aligned}
$$
obtain

$$
\begin{gathered}
F\left(x, \bar{x} ; \eta^{T}(x, \bar{x})\left(\sum_{i=1}^{k} \lambda_{i} \nabla f_{i}(\bar{x})+\sum_{j=1}^{m} \mu_{j} \nabla g_{j}(\bar{x})\right) P\right) \\
<-\left(\sum_{i=1}^{k} \frac{\lambda_{i} \rho_{i}}{\alpha_{i}(x, \bar{x})}+\sum_{j=1}^{m} \frac{\mu_{j} \tau_{j}}{\beta_{j}(x, \bar{x})}\right) d^{2}(x, \bar{x}) .
\end{gathered}
$$

From assumption (i), we have

$$
\left(\sum_{i=1}^{k} \frac{\lambda_{i} \rho_{i}}{\alpha_{i}(x, \bar{x})}+\sum_{j=1}^{m} \frac{\mu_{j} \tau_{j}}{\beta_{j}(x, \bar{x})}\right) d^{2}(x, \bar{x})<0 ;
$$

that is,

$$
\sum_{i=1}^{k} \frac{\lambda_{i} \rho_{i}}{\alpha_{i}(x, \bar{x})}+\sum_{j=1}^{m} \frac{\mu_{j} \tau_{j}}{\beta_{j}(x, \bar{x})}<0,
$$

which contradicts hypothesis (iii). That completes the proof.

Theorem 15. Let $\bar{x} \in R^{n}$ be a feasible solution in problem (MP). Suppose that

(i) there exists $\lambda_{i} \geqq 0, \sum_{i=1}^{k} \lambda_{i}=1, \mu_{j} \geqq 0, i \in K, j \in M$, such that

$$
\sum_{i=1}^{k} \lambda_{i} \nabla f_{i}(\bar{x})+\sum_{j=1}^{m} \mu_{j} \nabla g_{j}(\bar{x})=0 ;
$$

(ii) $(f, g)$ is pseudoquasi-invex $(F, P)$-type I at $\bar{x}$;

(iii) $\sum_{i=1}^{k}\left(\lambda_{i} \rho_{i} / \alpha_{i}(x, \bar{x})\right)+\sum_{j=1}^{m}\left(\mu_{j} \tau_{j} / \beta_{j}(x, \bar{x})\right) \geqq 0$.

Then $\bar{x}$ is a weakly efficient solution for (MP).

Proof. The proof follows the lines of Theorem 13.

Theorem 16. Let $\bar{x} \in R^{n}$ be a feasible solution in problem (MP). Suppose that

(i) there exists $\lambda_{i} \geqq 0, \sum_{i=1}^{k} \lambda_{i}=1, \mu_{j} \geqq 0, i \in K, j \in M$, such that

$$
\sum_{i=1}^{k} \lambda_{i} \nabla f_{i}(\bar{x})+\sum_{j=1}^{m} \mu_{j} \nabla g_{j}(\bar{x})=0
$$

(ii) $(f, g)$ is pseudoquasi-invex $(F, P)$-type II at $\bar{x}$;

(iii) $\sum_{i=1}^{k}\left(\lambda_{i} \rho_{i} / \alpha_{i}(x, \bar{x})\right)+\sum_{j=1}^{m}\left(\mu_{j} \tau_{j} / \beta_{j}(x, \bar{x})\right) \geqq 0$.

Then $\bar{x}$ is an efficient solution for (MP).

Proof. The proof follows the lines of Theorem 14.

Theorem 17. Let $\bar{x} \in R^{n}$ be a feasible solution in problem (MP). Suppose that

(i) there exists $\lambda_{i} \geqq 0, \sum_{i=1}^{k} \lambda_{i}=1, \mu_{j} \geqq 0$ (at least one $\left.\mu_{j}>0\right), i \in K, j \in M$, such that

$$
\sum_{i=1}^{k} \lambda_{i} \nabla f_{i}(\bar{x})+\sum_{j=1}^{m} \mu_{j} \nabla g_{j}(\bar{x})=0
$$

(ii) $(f, g)$ is quasipseudo-invex $(F, P)$-type I at $\bar{x}$;

(iii) $\sum_{i=1}^{k}\left(\lambda_{i} \rho_{i} / \alpha_{i}(x, \bar{x})\right)+\sum_{j=1}^{m}\left(\mu_{j} \tau_{j} / \beta_{j}(x, \bar{x})\right) \geqq 0$.

Then $\bar{x}$ is a weakly efficient solution for (MP).

Proof. Suppose contrary to the result that $\bar{x}$ is not a weakly efficient solution to (MP). Then there exists $x \in X_{0}$ such that

$$
f(x)<f(\bar{x}),
$$

with $b(x, \bar{x})>0$; the above inequality yields

$$
b(x, \bar{x})(f(x)-f(\bar{x}))<0,
$$

from hypothesis (ii), which implies

$$
\begin{aligned}
& F\left(x, \bar{x} ; \alpha_{i}(x, \bar{x}) \eta^{T}(x, \bar{x}) \nabla f_{i}(\bar{x}) P\right)+\rho_{i} d^{2}(x, \bar{x}) \leqq 0, \\
& i \in K, \\
& F\left(x, \bar{x} ; \beta_{j}(x, \bar{x}) \eta^{T}(x, \bar{x}) \nabla g_{j}(\bar{x}) P\right)+\tau_{j} d^{2}(x, \bar{x}) \\
& \quad<0, \quad j \in M .
\end{aligned}
$$


By sublinearity of $F$ with $\alpha_{i}(x, \bar{x})>0$ and $\beta_{j}(x, \bar{x})>0$, inequalities (43) yield

$$
\begin{array}{r}
F\left(x, \bar{x} ; \eta^{T}(x, \bar{x}) \nabla f_{i}(\bar{x}) P\right) \leqq-\frac{\rho_{i}}{\alpha_{i}(x, \bar{x})} d^{2}(x, \bar{x}), \\
i \in K, \\
F\left(x, \bar{x} ; \eta^{T}(x, \bar{x}) \nabla g_{j}(\bar{x}) P\right)<-\frac{\tau_{j}}{\beta_{j}(x, \bar{x})} d^{2}(x, \bar{x}), \\
j \in M .
\end{array}
$$

Using $\lambda_{i} \geqq 0, \sum_{i=1}^{k} \lambda_{i}=1$ along with the sublinearity of $F$, from inequality (44), we get

$$
\begin{aligned}
& F\left(x, \bar{x} ; \eta^{T}(x, \bar{x})\left(\sum_{i=1}^{k} \lambda_{i} \nabla f_{i}(\bar{x})\right) P\right) \\
& \leqq-\sum_{i=1}^{k} \frac{\lambda_{i} \rho_{i}}{\alpha_{i}(x, \bar{x})} d^{2}(x, \bar{x})
\end{aligned}
$$

With $\mu_{j} \geqq 0$ (at least one $\mu_{j}>0$ ), $j \in M$, and using the sublinearity of $F$, inequality (45) follows

$$
\begin{gathered}
F\left(x, \bar{x} ; \eta^{T}(x, \bar{x})\left(\sum_{j=1}^{m} \mu_{j} \nabla g_{j}(\bar{x})\right) P\right) \\
<-\sum_{j=1}^{m} \frac{\mu_{j} \tau_{j}}{\beta_{j}(x, \bar{x})} d^{2}(x, \bar{x}) .
\end{gathered}
$$

By the sublinearity of $F$, we sum (46) and (47) to obtain

$$
\begin{gathered}
F\left(x, \bar{x} ; \eta^{T}(x, \bar{x})\left(\sum_{i=1}^{k} \lambda_{i} \nabla f_{i}(\bar{x})+\sum_{j=1}^{m} \mu_{j} \nabla g_{j}(\bar{x})\right) P\right) \\
<-\left(\sum_{i=1}^{k} \frac{\lambda_{i} \rho_{i}}{\alpha_{i}(x, \bar{x})}+\sum_{j=1}^{m} \frac{\mu_{j} \tau_{j}}{\beta_{j}(x, \bar{x})}\right) d^{2}(x, \bar{x}),
\end{gathered}
$$

from hypothesis (iii), which follows

$$
F\left(x, \bar{x} ; \eta^{T}(x, \bar{x})\left(\sum_{i=1}^{k} \lambda_{i} \nabla f_{i}(\bar{x})+\sum_{j=1}^{m} \mu_{j} \nabla g_{j}(\bar{x})\right) P\right)
$$

$$
<0 .
$$

On the other hand, the hypothesis (i) implies

$$
\begin{aligned}
& F\left(x, \bar{x} ; \eta^{T}(x, \bar{x})\left(\sum_{i=1}^{k} \lambda_{i} \nabla f_{i}(\bar{x})+\sum_{j=1}^{m} \mu_{j} \nabla g_{j}(\bar{x})\right) P\right) \\
& \quad=0
\end{aligned}
$$

which contradicts (49). Hence the conclusion of theorem is established.
Example 18. We consider the following programming problem:

$$
\begin{array}{ll}
\text { Minimize } & f(x)=x^{3} \\
\text { subject to } & g(x)=1-e^{x} \leqq 0, \\
& x \in R .
\end{array}
$$

The set of all feasible solutions of $(\mathrm{P})$ can be given by $X_{0}=$ $\{x \mid x \geqq 0\}$.

Again, let $F(x, \bar{x} ; a)$ be the function defined by $F(x, \bar{x} ; a)=a(2 \bar{x}+1)$.

It can be verified that $(f, g)$ is quasipseudo-invex $(F, P)$ type I at $\bar{x}=0$ with $\alpha(x, \bar{x})=1 /\left(x^{2}+1\right), \beta(x, \bar{x})=2 /\left(x^{2}+\right.$ 1), $\eta(x, \bar{x})=x^{2}+1, P=1, d(x, \bar{x})=\bar{x}-1$, and $\rho=0$, $\tau=2 / 3, \nabla f(0)=0, \nabla g(0)=-1$.

Clearly, $\bar{x}=0$ is a feasible solution for problem (P) and it satisfied the assumptions of Theorem 17, as there exist $\lambda=$ $1, \mu=0$, such that

$$
\begin{aligned}
& \lambda \nabla f(0)+\mu \nabla g(0)=0, \\
& \frac{\lambda \rho}{\alpha(x, \bar{x})}+\frac{\mu \tau}{\beta(x, \bar{x})}=0 .
\end{aligned}
$$

We observe that there exists no other $x \in X_{0}$, such that $f(x)<f(\bar{x})$. Hence, $\bar{x}=0$ is an efficient solution for $(\mathrm{P})$.

Similarly, we can establish the following theorem.

Theorem 19. Let $\bar{x} \in R^{n}$ be a feasible solution in problem (MP). Suppose that

(i) there exists $\lambda_{i} \geqq 0, \sum_{i=1}^{k} \lambda_{i}=1, \mu_{j} \geqq 0$ (at least one $\mu_{j}>0$, as $j \in \bar{J}(\bar{x})$ ), $i \in K, j \in M$, such that

$$
\sum_{i=1}^{k} \lambda_{i} \nabla f_{i}(\bar{x})+\sum_{j=1}^{m} \mu_{j} \nabla g_{j}(\bar{x})=0
$$

(ii) $(f, g)$ is quasipseudo-invex $(F, P)$-type II at $\bar{x}$;

(iii) $\sum_{i=1}^{k}\left(\lambda_{i} \rho_{i} / \alpha_{i}(x, \bar{x})\right)+\sum_{j=1}^{m}\left(\mu_{j} \tau_{j} / \beta_{j}(x, \bar{x})\right) \geqq 0$.

Then $\bar{x}$ is an efficient solution for (MP).

\section{Mond-Weir Duality}

In this section, a dual problem is considered for the class of multiobjective programming problem with the new invex functions.

Consider the following Mond-Weir dual problem related to problem (MP): 
Maximize $f(u)=\left(f_{1}(u), f_{2}(u), \ldots, f_{k}(u)\right)$

subject to $\sum_{i=1}^{k} \bar{\lambda}_{i} \nabla f_{i}(u)+\sum_{j=1}^{m} \bar{\mu}_{j} \nabla g_{j}(u)=0$,

$$
\begin{aligned}
& \bar{\lambda}_{i} \geqq 0, \quad \sum_{i=1}^{k} \bar{\lambda}_{i}=1, \quad i \in K, \\
& \bar{\mu}_{j} \geqq 0, \quad j \in M .
\end{aligned}
$$

Let $W_{1}=\left\{(u, \bar{\lambda}, \bar{\mu}) \in X \times R^{k} \times R^{m}: \sum_{i=1}^{k} \bar{\lambda}_{i} \nabla f_{i}(u)+\right.$ $\left.\sum_{j=1}^{m} \bar{\mu}_{j} \nabla g_{j}(u)=0, \bar{\lambda} \geq 0, \sum_{i=1}^{k} \bar{\lambda}_{i}=1, \bar{\mu} \geqq 0\right\}$ be the set of all feasible solutions in problem (MDI).

Theorem 20 (weak duality). Let $x$ and $(u, \bar{\lambda}, \bar{\mu})$ be feasible solutions for (MP) and (MDI), respectively. Moreover, assume that

$$
\sum_{i=1}^{k} \frac{\bar{\lambda}_{i} \rho_{i}}{\alpha_{i}(x, u)}+\sum_{j=1}^{m} \frac{\bar{\mu}_{j} \tau_{j}}{\beta_{j}(x, u)} \geqq 0 .
$$

If one of the following conditions is satisfied:

(a) $(f, g)$ is pseudoinvex $(F, P)$-type I at $u$,

(b) $(f, g)$ is pseudoquasi-invex $(F, P)$-type I at $u$,

then the following can not hold:

$$
f(x)<f(u) .
$$

Proof. Suppose contrary to the result that

$$
f(x)<f(u)
$$

hold.

By $b(x, u)>0$, the above inequality follows

$$
b(x, u)(f(x)-f(u))<0 ;
$$

by assumption (a), $(f, g)$ is pseudoinvex $(F, P)$-type I at $u$, which yields

$$
\begin{aligned}
& F\left(x, u ; \alpha_{i}(x, u) \eta^{T}(x, u) \nabla f_{i}(u) P\right)+\rho_{i} d^{2}(x, u)<0, \\
& \quad i \in K, \\
& F\left(x, u ; \beta_{j}(x, u) \eta^{T}(x, u) \nabla g_{j}(u) P\right)+\tau_{j} d^{2}(x, u) \\
& \quad<0, \quad j \in M .
\end{aligned}
$$

By sublinearity of $F$ together with $\alpha_{i}(x, u)>0$ and $\beta_{j}(x, u)>0$, the above inequalities yield

$$
\begin{aligned}
& F\left(x, u ; \eta^{T}(x, u) \nabla f_{i}(u) P\right)<-\frac{\rho_{i}}{\alpha_{i}(x, u)} d^{2}(x, u), \\
& \quad i \in K, \\
& F\left(x, u ; \eta^{T}(x, u) \nabla g_{j}(u) P\right)<-\frac{\tau_{j}}{\beta_{j}(x, u)} d^{2}(x, u),
\end{aligned}
$$

From the feasibility of $(u, \bar{\lambda}, \bar{\mu})$ in Mond-Weir dual problem (MDI), it follows that $\bar{\lambda}_{i} \geqq 0, \sum_{i=1}^{k} \bar{\lambda}_{i}=1, \bar{\mu}_{j} \geqq 0$, $j \in M$. Multiplying inequalities (58) by $\bar{\lambda}_{i}$ and $\bar{\mu}_{j}$, respectively, together with the sublinearity of $F$, we get

$$
\begin{aligned}
& F\left(x, u ; \eta^{T}(x, u)\left(\sum_{i=1}^{k} \bar{\lambda}_{i} \nabla f_{i}(u)\right) P\right) \\
& <-\sum_{i=1}^{k} \frac{\bar{\lambda}_{i} \rho_{i}}{\alpha_{i}(x, u)} d^{2}(x, u), \\
& F\left(x, u ; \eta^{T}(x, u)\left(\sum_{j=1}^{m} \bar{\mu}_{j} \nabla g_{j}(u)\right) P\right) \\
& \leqq-\sum_{j=1}^{m} \frac{\bar{\mu}_{j} \tau_{j}}{\beta_{j}(x, u)} d^{2}(x, u) .
\end{aligned}
$$

Adding both sides of (59) with the sublinearity of $F$, we obtain

$$
\begin{gathered}
F\left(x, u ; \eta^{T}(x, u)\left(\sum_{i=1}^{k} \bar{\lambda}_{i} \nabla f_{i}(u)+\sum_{j=1}^{m} \bar{\mu}_{j} \nabla g_{j}(u)\right) P\right) \\
<-\left(\sum_{i=1}^{k} \frac{\bar{\lambda}_{i} \rho_{i}}{\alpha_{i}(x, u)}+\sum_{j=1}^{m} \frac{\bar{\mu}_{j} \tau_{j}}{\beta_{j}(x, u)}\right) d^{2}(x, u) .
\end{gathered}
$$

From assumption, $\sum_{i=1}^{k}\left(\bar{\lambda}_{i} \rho_{i} / \alpha_{i}(x, u)\right)+\sum_{j=1}^{m}\left(\bar{\mu}_{j} \tau_{j}\right)$ $\left.\beta_{j}(x, u)\right) \geqq 0$; inequality (60) implies

$$
F\left(x, u ; \eta^{T}(x, u)\left(\sum_{i=1}^{k} \bar{\lambda}_{i} \nabla f_{i}(u)+\sum_{j=1}^{m} \bar{\mu}_{j} \nabla g_{j}(u)\right) P\right)
$$

$<0$.

By the constraint condition of dual problem (MDI) and $F(x, u ; 0)=0$, we have

$$
\begin{aligned}
& F\left(x, u ; \eta^{T}(x, u)\left(\sum_{i=1}^{k} \bar{\lambda}_{i} \nabla f_{i}(u)+\sum_{j=1}^{m} \bar{\mu}_{j} \nabla g_{j}(u)\right) P\right) \\
& \quad=0
\end{aligned}
$$

which contradicts inequality (61). Thus, the conclusion of theorem holds.

The proof of part (b) is similar to the proof of part (a).

Theorem 21 (weak duality). Let $x$ and $(u, \bar{\lambda}, \bar{\mu})$ be feasible solutions for (MP) and (MDI), respectively. Moreover, assume that

$$
\sum_{i=1}^{k} \frac{\bar{\lambda}_{i} \rho_{i}}{\alpha_{i}(x, u)}+\sum_{j=1}^{m} \frac{\bar{\mu}_{j} \tau_{j}}{\beta_{j}(x, u)} \geqq 0 .
$$

If one of the following conditions is satisfied:

(a) $(f, g)$ is pseudoinvex $(F, P)$-type II at $u$, 
(b) $(f, g)$ is pseudoquasi-invex $(F, P)$-type II at $u$,

then the following can not hold:

$$
f(x) \leq f(u) .
$$

Proof. Suppose contrary to the result that

$$
f(x) \leq f(u)
$$

hold.

By $b(x, u)>0$, the above inequality yields

$$
b(x, u)(f(x)-f(u)) \leq 0 ;
$$

by assumption (a), $(f, g)$ is pseudoinvex $(F, P)$-type II at $u$, which yields

$$
\begin{aligned}
& F\left(x, u ; \alpha_{i}(x, u) \eta^{T}(x, u) \nabla f_{i}(u) P\right)+\rho_{i} d^{2}(x, u)<0, \\
& i \in K, \\
& F\left(x, u ; \beta_{j}(x, u) \eta^{T}(x, u) \nabla g_{j}(u) P\right)+\tau_{j} d^{2}(x, u) \\
& \quad<0, \quad j \in M .
\end{aligned}
$$

By sublinearity of $F$ together with $\alpha_{i}(x, u)>0$ and $\beta_{j}(x, u)>0$, the above inequalities yield

$$
\begin{array}{r}
F\left(x, u ; \eta^{T}(x, u) \nabla f_{i}(u) P\right)<-\frac{\rho_{i}}{\alpha_{i}(x, u)} d^{2}(x, u), \\
i \in K, \\
F\left(x, u ; \eta^{T}(x, u) \nabla g_{j}(u) P\right)<-\frac{\tau_{j}}{\beta_{j}(x, u)} d^{2}(x, u), \\
\quad j \in M .
\end{array}
$$

From the feasibility of $(u, \bar{\lambda}, \bar{\mu})$ in Mond-Weir dual problem (MDI), we have

$$
\sum_{i=1}^{k} \bar{\lambda}_{i} \nabla f_{i}(u)+\sum_{j=1}^{m} \bar{\mu}_{j} \nabla g_{j}(u)=0 .
$$

For $\bar{\lambda}_{i} \geqq 0, \sum_{i=1}^{k} \bar{\lambda}_{i}=1, \bar{\mu}_{j} \geqq 0, j \in M$. Multiplying inequalities (68) by $\bar{\lambda}_{i}$ and $\bar{\mu}_{j}$, respectively, together with the sublinearity of $F$, we have

$$
\begin{aligned}
& F\left(x, u ; \eta^{T}(x, u)\left(\sum_{i=1}^{k} \bar{\lambda}_{i} \nabla f_{i}(u)\right) P\right) \\
& <-\sum_{i=1}^{k} \frac{\bar{\lambda}_{i} \rho_{i}}{\alpha_{i}(x, u)} d^{2}(x, u), \\
& F\left(x, u ; \eta^{T}(x, u)\left(\sum_{j=1}^{m} \bar{\mu}_{j} \nabla g_{j}(u)\right) P\right) \\
& \leqq-\sum_{j=1}^{m} \frac{\bar{\mu}_{j} \tau_{j}}{\beta_{j}(x, u)} d^{2}(x, u) .
\end{aligned}
$$

Adding both sides of (70) with the sublinearity of $F$, we get

$$
\begin{gathered}
F\left(x, u ; \eta^{T}(x, u)\left(\sum_{i=1}^{k} \bar{\lambda}_{i} \nabla f_{i}(u)+\sum_{j=1}^{m} \bar{\mu}_{j} \nabla g_{j}(u)\right) P\right) \\
<-\left(\sum_{i=1}^{k} \frac{\bar{\lambda}_{i} \rho_{i}}{\alpha_{i}(x, u)}+\sum_{j=1}^{m} \frac{\bar{\mu}_{j} \tau_{j}}{\beta_{j}(x, u)}\right) d^{2}(x, u) .
\end{gathered}
$$

Combining (69) and (71), we obtain

$$
\sum_{i=1}^{k} \frac{\bar{\lambda}_{i} \rho_{i}}{\alpha_{i}(x, u)}+\sum_{j \in \bar{J}(u)} \frac{\bar{\mu}_{j} \tau_{j}}{\beta_{j}(x, u)}<0,
$$

which contradicts the assumption

$$
\sum_{i=1}^{k} \frac{\bar{\lambda}_{i} \rho_{i}}{\alpha_{i}(x, u)}+\sum_{j \in \bar{J}(u)} \frac{\bar{\mu}_{j} \tau_{j}}{\beta_{j}(x, u)} \geqq 0 .
$$

Thus, the conclusion of the theorem holds.

The proof of part (b) is similar to the proof of part (a).

Theorem 22 (strong duality). Assume that $\bar{x}$ is a weakly efficient solution of (MP). Suppose that there exists $\bar{\lambda} \geq 0$ and $\bar{\mu} \geqq 0$, such that $(\bar{x}, \bar{\lambda}, \bar{\mu})$ is feasible for (MDI). Furthermore, if the weak duality Theorem 20 holds for all feasible solutions of the problems (MP) and (MDI), then $(\bar{x}, \bar{\lambda}, \bar{\mu})$ is a weakly efficient solution of (MDI).

Proof. Suppose that $(\bar{x}, \bar{\lambda}, \bar{\mu})$ is not a weakly efficient solution of (MDI); then there exists another feasible solution $(u, \lambda, \mu)$ of (MDI) such that

$$
f(\bar{x})<f(u),
$$

which is a contradiction to Theorem 20 . Hence $(\bar{x}, \bar{\lambda}, \bar{\mu})$ is a weakly efficient solution of (MDI).

Theorem 23 (strong duality). Assume that $\bar{x}$ is an efficient solution of (MP). Suppose that there exist $\bar{\lambda} \geq 0$ and $\bar{\mu} \geqq 0$, such that $(\bar{x}, \bar{\lambda}, \bar{\mu})$ is feasible for (MDI). Furthermore, if the weak duality Theorem 21 holds for all feasible solutions of the problems (MP) and (MDI), then $(\bar{x}, \bar{\lambda}, \bar{\mu})$ is an efficient solution of $(\mathrm{MDI})$.

Proof. Suppose that $(\bar{x}, \bar{\lambda}, \bar{\mu})$ is not an efficient solution of (MDI); then there exists another feasible solution $(u, \lambda, \mu)$ of (MDI) such that

$$
f(\bar{x}) \leq f(u),
$$

which is a contradiction to Theorem 21 . Hence $(\bar{x}, \bar{\lambda}, \bar{\mu})$ is an efficient solution of (MDI).

Theorem 24 (strict converse duality). Let $\bar{x}$ and $(\bar{u}, \bar{\lambda}, \bar{\mu})$ be feasible solutions for (MP) and (MDI), respectively. Suppose that $f(\bar{x}) \leq f(\bar{u})$, and $\sum_{i=1}^{k}\left(\bar{\lambda}_{i} \rho_{i} / \alpha_{i}(\bar{x}, \bar{u})\right)+\sum_{j=1}^{m}\left(\bar{\mu}_{j} \tau_{j} /\right.$ $\left.\beta_{j}(\bar{x}, \bar{u})\right) \geqq 0$. If one of the following conditions is satisfied: 
(a) $(f, g)$ is pseudoinvex $(F, P)$-type II at $\bar{u}$,

(b) $(f, g)$ is pseudoquasi-invex $(F, P)$-type II at $\bar{u}$,

then $\bar{x}=\bar{u}$.

Proof. Suppose that $\bar{x} \neq \bar{u}$.

By $b(\bar{x}, \bar{u})>0$, the condition $f(\bar{x}) \leq f(\bar{u})$ yields

$$
b(\bar{x}, \bar{u})(f(\bar{x})-f(\bar{u})) \leq 0,
$$

using assumption $(\mathrm{a}),(f, g)$ is pseudoinvex $(F, P)$-type II at $\bar{u}$, which yields

$$
\begin{aligned}
& F\left(\bar{x}, \bar{u} ; \alpha_{i}(\bar{x}, \bar{u}) \eta^{T}(\bar{x}, \bar{u}) \nabla f_{i}(\bar{u}) P\right)+\rho_{i} d^{2}(\bar{x}, \bar{u})<0, \\
& i \in K, \\
& F\left(\bar{x}, \bar{u} ; \beta_{j}(\bar{x}, \bar{u}) \eta^{T}(\bar{x}, \bar{u}) \nabla g_{j}(\bar{u}) P\right)+\tau_{j} d^{2}(\bar{x}, \bar{u}) \\
& \quad<0, \quad j \in M .
\end{aligned}
$$

By sublinearity of $F$ together with $\alpha_{i}(\bar{x}, \bar{u})>0$ and $\beta_{j}(\bar{x}, \bar{u})>0$, the above inequalities yield

$$
\begin{gathered}
F\left(\bar{x}, \bar{u} ; \eta^{T}(\bar{x}, \bar{u}) \nabla f_{i}(\bar{u}) P\right)<-\frac{\rho_{i}}{\alpha_{i}(\bar{x}, \bar{u})} d^{2}(\bar{x}, \bar{u}), \\
i \in K, \\
F\left(\bar{x}, \bar{u} ; \eta^{T}(\bar{x}, \bar{u}) \nabla g_{j}(\bar{u}) P\right)<-\frac{\tau_{j}}{\beta_{j}(\bar{x}, \bar{u})} d^{2}(\bar{x}, \bar{u}),
\end{gathered}
$$$$
j \in M \text {. }
$$

Because $(\overline{\mathcal{u}}, \bar{\lambda}, \bar{\mu})$ is feasible for (MDI), then

$$
\sum_{i=1}^{k} \bar{\lambda}_{i} \nabla f_{i}(\bar{u})+\sum_{j=1}^{m} \bar{\mu}_{j} \nabla g_{j}(\bar{u})=0 .
$$

For $\bar{\lambda}_{i} \geqq 0, \sum_{i=1}^{k} \bar{\lambda}_{i}=1, \bar{\mu}_{j} \geqq 0, j \in M$. Multiplying inequalities (78) by $\bar{\lambda}_{i}$ and $\bar{\mu}_{j}$, respectively, together with the sublinearity of $F$, we have

$$
\begin{aligned}
& F\left(\bar{x}, \bar{u} ; \eta^{T}(\bar{x}, \bar{u})\left(\sum_{i=1}^{k} \bar{\lambda}_{i} \nabla f_{i}(\bar{u})\right) P\right) \\
& <-\sum_{i=1}^{k} \frac{\bar{\lambda}_{i} \rho_{i}}{\alpha_{i}(\bar{x}, \bar{u})} d^{2}(\bar{x}, \bar{u}), \\
& F\left(\bar{x}, \bar{u} ; \eta^{T}(\bar{x}, \bar{u})\left(\sum_{j=1}^{m} \bar{\mu}_{j} \nabla g_{j}(\bar{u})\right) P\right) \\
& \leqq-\sum_{j=1}^{m} \frac{\bar{\mu}_{j} \tau_{j}}{\beta_{j}(\bar{x}, \bar{u})} d^{2}(\bar{x}, \bar{u}) .
\end{aligned}
$$

Adding both sides of (80) with the sublinearity of $F$, we get

$$
\begin{gathered}
F\left(\bar{x}, \bar{u} ; \eta^{T}(\bar{x}, \bar{u})\left(\sum_{i=1}^{k} \bar{\lambda}_{i} \nabla f_{i}(\bar{u})+\sum_{j=1}^{m} \bar{\mu}_{j} \nabla g_{j}(\bar{u})\right) P\right) \\
<-\left(\sum_{i=1}^{k} \frac{\bar{\lambda}_{i} \rho_{i}}{\alpha_{i}(\bar{x}, \bar{u})}+\sum_{j=1}^{m} \frac{\bar{\mu}_{j} \tau_{j}}{\beta_{j}(\bar{x}, \bar{u})}\right) d^{2}(\bar{x}, \bar{u}) .
\end{gathered}
$$

Using the assumption $\sum_{i=1}^{k}\left(\bar{\lambda}_{i} \rho_{i} / \alpha_{i}(\bar{x}, \bar{u})\right)+\sum_{j=1}^{m}\left(\bar{\mu}_{j} \tau_{j} /\right.$ $\left.\beta_{j}(\bar{x}, \bar{u})\right) \geqq 0$, inequality (81) follows that

$$
\begin{aligned}
& F\left(\bar{x}, \bar{u} ; \eta^{T}(\bar{x}, \bar{u})\left(\sum_{i=1}^{k} \bar{\lambda}_{i} \nabla f_{i}(\bar{u})+\sum_{j=1}^{m} \bar{\mu}_{j} \nabla g_{j}(\bar{u})\right) P\right) \\
& \quad<0 .
\end{aligned}
$$

Using inequality (79) together with the sublinearity of $F$, we obtain

$$
\begin{aligned}
& F\left(\bar{x}, \bar{u} ; \eta^{T}(\bar{x}, \bar{u})\left(\sum_{i=1}^{k} \bar{\lambda}_{i} \nabla f_{i}(\bar{u})+\sum_{j=1}^{m} \bar{\mu}_{j} \nabla g_{j}(\bar{u})\right) P\right) \\
& \quad=0
\end{aligned}
$$

which is a contradiction to (82). Then $\bar{x}=\bar{u}$.

The proof of part (b) is similar to the proof of part (a).

Theorem 25 (strict converse duality). Let $\bar{x}$ and $(\bar{u}, \bar{\lambda}, \bar{\mu})$ be feasible solutions for (MP) and (MDI), respectively. Suppose that $f(\bar{x})<f(\bar{u})$, and $\sum_{i=1}^{k}\left(\bar{\lambda}_{i} \rho_{i} / \alpha_{i}(\bar{x}, \bar{u})\right)+\sum_{j=1}^{m}\left(\bar{\mu}_{j} \tau_{j} /\right.$ $\left.\beta_{j}(\bar{x}, \bar{u})\right) \geqq 0$. If one of the following conditions is satisfied:

(a) $(f, g)$ is pseudoinvex $(F, P)$-type I at $\bar{u}$,

(b) $(f, g)$ is pseudoquasi-invex $(F, P)$-type I at $\bar{u}$,

then $\bar{x}=\bar{u}$.

Proof. Suppose that $\bar{x} \neq \bar{u}$.

By $b(\bar{x}, \bar{u})>0$, the condition $f(\bar{x})<f(\bar{u})$ yields

$$
b(\bar{x}, \bar{u})(f(\bar{x})-f(\bar{u}))<0 ;
$$

using assumption (a), $(f, g)$ is pseudoinvex $(F, P)$-type I at $\bar{u}$, which yields

$$
\begin{aligned}
& F\left(\bar{x}, \bar{u} ; \alpha_{i}(\bar{x}, \bar{u}) \eta^{T}(\bar{x}, \bar{u}) \nabla f_{i}(\bar{u}) P\right)+\rho_{i} d^{2}(\bar{x}, \bar{u})<0, \\
& i \in K, \\
& F\left(\bar{x}, \bar{u} ; \beta_{j}(\bar{x}, \bar{u}) \eta^{T}(\bar{x}, \bar{u}) \nabla g_{j}(\bar{u}) P\right)+\tau_{j} d^{2}(\bar{x}, \bar{u}) \\
& \quad<0, \quad j \in M .
\end{aligned}
$$


By using the sublinearity of $F$ with $\alpha_{i}(\bar{x}, \bar{u})>0$ and $\beta_{j}(\bar{x}$, $\bar{u})>0$ and $(\bar{u}, \bar{\lambda}, \bar{\mu}) \in W_{1}$, inequalities (85) imply

$$
\begin{aligned}
& F\left(\bar{x}, \bar{u} ; \eta^{T}(\bar{x}, \bar{u})\left(\sum_{i=1}^{k} \bar{\lambda}_{i} \nabla f_{i}(\bar{u})\right) P\right) \\
& <-\sum_{i=1}^{k} \frac{\bar{\lambda}_{i} \rho_{i}}{\alpha_{i}(\bar{x}, \bar{u})} d^{2}(\bar{x}, \bar{u}), \\
& F\left(\bar{x}, \bar{u} ; \eta^{T}(\bar{x}, \bar{u})\left(\sum_{j=1}^{m} \bar{\mu}_{j} \nabla g_{j}(\bar{u})\right) P\right) \\
& \leqq-\sum_{j=1}^{m} \frac{\bar{\mu}_{j} \tau_{j}}{\beta_{j}(\bar{x}, \bar{u})} d^{2}(\bar{x}, \bar{u}) .
\end{aligned}
$$
have

Adding both sides of (86) with the sublinearity of $F$, we

$$
\begin{gathered}
F\left(\bar{x}, \bar{u} ; \eta^{T}(\bar{x}, \bar{u})\left(\sum_{i=1}^{k} \bar{\lambda}_{i} \nabla f_{i}(\bar{u})+\sum_{j=1}^{m} \bar{\mu}_{j} \nabla g_{j}(\bar{u})\right) P\right) \\
<-\left(\sum_{i=1}^{k} \frac{\bar{\lambda}_{i} \rho_{i}}{\alpha_{i}(\bar{x}, \bar{u})}+\sum_{j=1}^{m} \frac{\bar{\mu}_{j} \tau_{j}}{\beta_{j}(\bar{x}, \bar{u})}\right) d^{2}(\bar{x}, \bar{u}) .
\end{gathered}
$$

Using the condition $\sum_{i=1}^{k}\left(\bar{\lambda}_{i} \rho_{i} / \alpha_{i}(\bar{x}, \bar{u})\right)+\sum_{j=1}^{m}\left(\bar{\mu}_{j} \tau_{j} /\right.$ $\left.\beta_{j}(\bar{x}, \bar{u})\right) \geqq 0$, inequality $(87)$ yields

$$
\begin{aligned}
& F\left(\bar{x}, \bar{u} ; \eta^{T}(\bar{x}, \bar{u})\left(\sum_{i=1}^{k} \bar{\lambda}_{i} \nabla f_{i}(\bar{u})+\sum_{j=1}^{m} \bar{\mu}_{j} \nabla g_{j}(\bar{u})\right) P\right) \\
& \quad<0 .
\end{aligned}
$$

According to the constraint condition of (MDI) with $F(\bar{x}, \bar{u} ; 0)=0$, we get

$$
\begin{aligned}
& F\left(\bar{x}, \bar{u} ; \eta^{T}(\bar{x}, \bar{u})\left(\sum_{i=1}^{k} \bar{\lambda}_{i} \nabla f_{i}(\bar{u})+\sum_{j=1}^{m} \bar{\mu}_{j} \nabla g_{j}(\bar{u})\right) P\right) \\
& \quad=0
\end{aligned}
$$

which is a contradiction to (88). Then $\bar{x}=\bar{u}$.

The proof of part (b) is similar to the proof of part (a).

\section{Wolfe Type Duality}

In this section, we consider the Wolfe type dual for (MP) and establish various duality theorems. Let $e$ be the vector of $R^{k}$ whose components are all ones.

$$
\begin{array}{ll}
\text { Maximize } & G(u)=f(u)+\bar{\mu}^{T} g(u) e=\left(f_{1}(u)+\sum_{j=1}^{m} \bar{\mu}_{j} g_{j}(u), f_{2}(u)+\sum_{j=1}^{m} \bar{\mu}_{j} g_{j}(u), \ldots, f_{k}(u)+\sum_{j=1}^{m} \bar{\mu}_{j} g_{j}(u)\right) \\
\text { subject to } & \sum_{i=1}^{k} \bar{\lambda}_{i} \nabla f_{i}(u)+\sum_{j=1}^{m} \bar{t}_{j} \nabla g_{j}(u)=0, \\
& \bar{t}_{j}=2 \bar{\mu}_{j}, \quad j \in M, \\
& \bar{\lambda}_{i} \geqq 0, \quad \sum_{i=1}^{k} \bar{\lambda}_{i}=1, i \in K, \\
& \bar{\mu}_{j} \geqq 0, \quad j \in M .
\end{array}
$$

Let $W_{2}=\left\{(u, \bar{\lambda}, \bar{\mu}, \bar{t}) \in X \times R^{k} \times R^{m} \times R^{m}: \sum_{i=1}^{k} \bar{\lambda}_{i} \nabla f_{i}(u)+\right.$ $\left.\sum_{j=1}^{m} \bar{t}_{j} g_{j}(u)=0, \bar{\lambda} \geq 0, \sum_{i=1}^{k} \bar{\lambda}_{i}=1, \bar{\mu} \geqq 0, \bar{t} \geqq 0\right\}$ be the set of all feasible solutions in problem (MDII).

Theorem 26 (weak duality). Let $x \in X_{0}$ and $(u, \bar{\lambda}, \bar{\mu}, \bar{t}) \in W_{2}$. Suppose that

$$
\sum_{i=1}^{k} \frac{\bar{\lambda}_{i} \rho_{i}}{\alpha_{i}(x, u)}+\sum_{j=1}^{m} \frac{\bar{\mu}_{j} \tau_{j}}{\beta_{j}(x, u)} \geqq 0 .
$$

If one of the following conditions is satisfied:

(a) $(G, g)$ is pseudoinvex $(F, P)$-type I at $u$,

(b) $(G, g)$ is pseudoquasi-invex $(F, P)$-type I at $u$, then the following can not hold:

$$
f(x)<G(u) .
$$

Proof. If

$$
f(x)<G(u)
$$

holds, then we have

$$
f(x)<f(u)+\bar{\mu}^{T} g(u) e .
$$

Since $x \in X_{0}$ and $\bar{\mu} \geqq 0$, the above inequality yields

$$
f(x)+\bar{\mu}^{T} g(x) e<f(u)+\bar{\mu}^{T} g(u) e ;
$$


that is,

$$
G(x)<G(u)
$$

by using $b(x, u)>0$, we have

$$
b(x, u)(G(x)-G(u))<0 \text {; }
$$

by assumption (a), $(G, g)$ is pseudoinvex $(F, P)$-type I at $u$, which implies

$$
\begin{aligned}
& F\left(x, u ; \alpha_{i}(x, u) \eta^{T}(x, u)\left(\nabla f_{i}(u)+\sum_{j=1}^{m} \bar{\mu}_{j} \nabla g_{j}(u)\right)\right. \\
& \cdot P)+\rho_{i} d^{2}(x, u)<0, \quad i \in K, \\
& F\left(x, u ; \beta_{j}(x, u) \eta^{T}(x, u) \nabla g_{j}(u) P\right)+\tau_{j} d^{2}(x, u) \\
& \quad<0, \quad j \in M .
\end{aligned}
$$

By sublinearity of $F$ together with $\alpha_{i}(x, u)>0, \beta_{j}(x, u)>$ 0 , and the feasibility of $(u, \bar{\lambda}, \bar{\mu}, \bar{t})$ in (MDII), the above inequalities yield

$$
\begin{aligned}
& F\left(x, u ; \eta^{T}(x, u)\left(\sum_{i=1}^{k} \bar{\lambda}_{i} \nabla f_{i}(u)+\sum_{j=1}^{m} \bar{\mu}_{j} \nabla g_{j}(u)\right) P\right) \\
& <-\sum_{i=1}^{k} \frac{\bar{\lambda}_{i} \rho_{i}}{\alpha_{i}(x, u)} d^{2}(x, u), \\
& F\left(x, u ; \eta^{T}(x, u)\left(\sum_{j=1}^{m} \bar{\mu}_{j} \nabla g_{j}(u)\right) P\right) \\
& \leqq-\sum_{j=1}^{m} \frac{\bar{\mu}_{j} \tau_{j}}{\beta_{j}(x, u)} d^{2}(x, u) .
\end{aligned}
$$

Adding both sides of (98) with the sublinearity of $F$, we get

$$
\begin{array}{r}
F\left(x, u ; \eta^{T}(x, u)\left(\sum_{i=1}^{k} \bar{\lambda}_{i} \nabla f_{i}(u)+\sum_{j=1}^{m} \bar{t}_{j} \nabla g_{j}(u)\right) P\right) \\
<-\left(\sum_{i=1}^{k} \frac{\bar{\lambda}_{i} \rho_{i}}{\alpha_{i}(x, u)}+\sum_{j=1}^{m} \frac{\bar{\mu}_{j} \tau_{j}}{\beta_{j}(x, u)}\right) d^{2}(x, u), \\
\left(\bar{t}_{j}=2 \bar{\mu}_{j}, j \in M\right) .
\end{array}
$$

From assumption, $\sum_{i=1}^{k}\left(\bar{\lambda}_{i} \rho_{i} / \alpha_{i}(x, u)\right)+\sum_{j=1}^{m}\left(\bar{\mu}_{j} \tau_{j} / \beta_{j}(x\right.$, $u)) \geqq 0$; inequality (99) implies

$$
\begin{aligned}
& F\left(x, u ; \eta^{T}(x, u)\left(\sum_{i=1}^{k} \bar{\lambda}_{i} \nabla f_{i}(u)+\sum_{j=1}^{m} \bar{t}_{j} \nabla g_{j}(u)\right) P\right) \\
& \quad<0 .
\end{aligned}
$$

By the constraint condition of (MDII) and $F(x, u ; 0)=0$, we have

$$
\begin{aligned}
& F\left(x, u ; \eta^{T}(x, u)\left(\sum_{i=1}^{k} \bar{\lambda}_{i} \nabla f_{i}(u)+\sum_{j=1}^{m} \bar{t}_{j} \nabla g_{j}(u)\right) P\right) \\
& \quad=0,
\end{aligned}
$$

which contradicts inequality (100). Thus, the conclusion of theorem holds.

The proof of part (b) is similar to the proof of part (a).

Theorem 27 (weak duality). Let $x \in X_{0}$ and $(u, \bar{\lambda}, \bar{\mu}, \bar{t}) \in W_{2}$. Suppose that

$$
\sum_{i=1}^{k} \frac{\bar{\lambda}_{i} \rho_{i}}{\alpha_{i}(x, u)}+\sum_{j=1}^{m} \frac{\bar{\mu}_{j} \tau_{j}}{\beta_{j}(x, u)} \geqq 0 .
$$

If one of the following conditions is satisfied:

(a) $(G, g)$ is pseudoinvex $(F, P)$-type II at $u$,

(b) $(G, g)$ is pseudoquasi-invex $(F, P)$-type II at $u$, then the following can not hold:

$$
f(x) \leq G(u) .
$$

Proof. Suppose contrary to the result that

$$
f(x) \leq G(u)
$$

hold. That is,

$$
f(x) \leq f(u)+\bar{\mu}^{T} g(u) e .
$$

Since $x \in X_{0}$ and $\bar{\mu} \geqq 0$, the above inequality yields

$$
f(x)+\bar{\mu}^{T} g(x) e \leq f(u)+\bar{\mu}^{T} g(u) e ;
$$

that is,

$$
G(x) \leq G(u) .
$$

By using $b(x, u)>0$, we get

$$
b(x, u)(G(x)-G(u)) \leq 0 .
$$

By assumption (a), $(G, g)$ is pseudoinvex $(F, P)$-type II at $u$, which yields

$$
\begin{aligned}
& F\left(x, u ; \alpha_{i}(x, u) \eta^{T}(x, u)\left(\nabla f_{i}(u)+\sum_{j=1}^{m} \bar{\mu}_{j} \nabla g_{j}(u)\right)\right. \\
& \cdot P)+\rho_{i} d^{2}(x, u)<0, \quad i \in K, \\
& F\left(x, u ; \beta_{j}(x, u) \eta^{T}(x, u) \nabla g_{j}(u) P\right)+\tau_{j} d^{2}(x, u) \\
& \quad<0, \quad j \in M .
\end{aligned}
$$


By sublinearity of $F$ together with $\alpha_{i}(x, u)>0, \beta_{j}(x, u)>$ 0 , and the feasibility of $(u, \bar{\lambda}, \bar{\mu}, \bar{t})$ in (MDII), the above inequalities yield

$$
\begin{aligned}
& F\left(x, u ; \eta^{T}(x, u)\left(\sum_{i=1}^{k} \bar{\lambda}_{i} \nabla f_{i}(u)+\sum_{j=1}^{m} \bar{\mu}_{j} \nabla g_{j}(u)\right) P\right) \\
& <-\sum_{i=1}^{k} \frac{\bar{\lambda}_{i} \rho_{i}}{\alpha_{i}(x, u)} d^{2}(x, u), \\
& F\left(x, u ; \eta^{T}(x, u)\left(\sum_{j=1}^{m} \bar{\mu}_{j} \nabla g_{j}(u)\right) P\right) \\
& \quad \leqq-\sum_{j=1}^{m} \frac{\bar{\mu}_{j} \tau_{j}}{\beta_{j}(x, u)} d^{2}(x, u) .
\end{aligned}
$$
get

Adding both sides of (110) with the sublinearity of $F$, we

$$
\begin{array}{r}
F\left(x, u ; \eta^{T}(x, u)\left(\sum_{i=1}^{k} \bar{\lambda}_{i} \nabla f_{i}(u)+\sum_{j=1}^{m} \bar{t}_{j} \nabla g_{j}(u)\right) P\right) \\
<-\left(\sum_{i=1}^{k} \frac{\bar{\lambda}_{i} \rho_{i}}{\alpha_{i}(x, u)}+\sum_{j=1}^{m} \frac{\bar{\mu}_{j} \tau_{j}}{\beta_{j}(x, u)}\right) d^{2}(x, u), \\
\left(\bar{t}_{j}=2 \bar{\mu}_{j}, j \in M\right) .
\end{array}
$$

By using the constraint condition of (MDII) and $F(x$, $u ; 0)=0$, inequality (111) implies

$$
\sum_{i=1}^{k} \frac{\bar{\lambda}_{i} \rho_{i}}{\alpha_{i}(x, u)}+\sum_{j=1}^{m} \frac{\bar{\mu}_{j} \tau_{j}}{\beta_{j}(x, u)}<0,
$$

which contradicts the assumption $\sum_{i=1}^{k}\left(\bar{\lambda}_{i} \rho_{i} / \alpha_{i}(x, u)\right)+$ $\sum_{j=1}^{m}\left(\bar{\mu}_{j} \tau_{j} / \beta_{j}(x, u)\right) \geqq 0$.

Thus, the conclusion of theorem holds.

The proof of part (b) is similar to the proof of part (a).

Theorem 28 (strong duality). Assume that $\bar{x}$ is a weakly efficient solution of (MP). Suppose that there exist $\bar{\lambda} \geq 0$, $\bar{\mu} \geqq 0$, and $\bar{t} \geqq 0$, such that $(\bar{x}, \bar{\lambda}, \bar{\mu}, \bar{t})$ is feasible for (MDII). Furthermore, if the weak duality Theorem 26 holds for all feasible solutions of the problems (MP) and (MDII), then $(\bar{x}, \bar{\lambda}, \bar{\mu}, \bar{t})$ is a weakly efficient solution of (MDII).

Proof. Suppose that $(\bar{x}, \bar{\lambda}, \bar{\mu}, \bar{t})$ is not a weakly efficient solution of (MDII); then there exists another feasible solution $(x, \lambda, \mu, t)$ of (MDII) such that

$$
G(\bar{x})<G(u) \text {; }
$$

that is,

$$
f(\bar{x})+\bar{\mu}^{T} g(\bar{x}) e<f(u)+\mu^{T} g(u) e .
$$

For $\bar{x} \in X_{0}$ and $(\bar{x}, \bar{\lambda}, \bar{\mu}, \bar{t}) \in W_{2}$, the above inequality yields

$$
f(\bar{x})<f(u)+\mu^{T} g(u) e ;
$$

that is,

$$
f(\bar{x})<G(u),
$$

which is a contradiction to Theorem 26 . Hence $(\bar{x}, \bar{\lambda}, \bar{\mu}, \bar{t})$ is a weakly efficient solution of (MDII).

Theorem 29 (strict converse duality). Let $\bar{x}$ and $(\bar{u}, \bar{\lambda}, \bar{\mu}, \bar{t})$ be feasible solutions for (MP) and (MDII), respectively. Suppose that $f(\bar{x})<G(\bar{u})$, and $\sum_{i=1}^{k}\left(\bar{\lambda}_{i} \rho_{i} / \alpha_{i}(\bar{x}, \bar{u})\right)+$ $\sum_{j=1}^{m}\left(\bar{\mu}_{j} \tau_{j} / \beta_{j}(\bar{x}, \bar{u})\right) \geqq 0$. If one of the following conditions is satisfied:

(a) $(f, g)$ is pseudoinvex $(F, P)$-type I at $\bar{u}$,

(b) $(f, g)$ is pseudoquasi-invex $(F, P)$-type I at $\bar{u}$,

then $\bar{x}=\bar{u}$.

Proof. Suppose that $\bar{x} \neq \bar{u}$.

From $\bar{x} \in X_{0}$ and $(\bar{u}, \bar{\lambda}, \bar{\mu}, \bar{t}) \in W_{2}$, the condition $f(\bar{x})<$ $G(\bar{u})$ yields

$$
f(\bar{x})+\bar{\mu}^{T} g(\bar{x}) e<f(\bar{u})+\bar{\mu}^{T} g(\bar{u}) e
$$

that is,

$$
G(\bar{x})<G(\bar{u}),
$$

by $b(\bar{x}, \bar{u})>0$, which implies

$$
b(\bar{x}, \bar{u})(G(\bar{x})-G(\bar{u}))<0
$$

using assumption (a), $(G, g)$ is pseudoinvex $(F, P)$-type I at $\bar{u}$, which yields

$$
\begin{aligned}
& F\left(\bar{x}, \bar{u} ; \alpha_{i}(\bar{x}, \bar{u}) \eta^{T}(\bar{x}, \bar{u})\left(\nabla f_{i}(\bar{u})+\sum_{j=1}^{m} \bar{\mu}_{j} \nabla g_{j}(\bar{u})\right)\right. \\
& \cdot P)+\rho_{i} d^{2}(\bar{x}, \bar{u})<0, \quad i \in K ; \\
& F\left(\bar{x}, \bar{u} ; \beta_{j}(\bar{x}, \bar{u}) \eta^{T}(\bar{x}, \bar{u}) \nabla g_{j}(\bar{u}) P\right)+\tau_{j} d^{2}(\bar{x}, \bar{u}) \\
& \quad<0, \quad j \in M .
\end{aligned}
$$


By sublinearity of $F$ together with $\alpha_{i}(\bar{x}, \bar{u})>0, \beta_{j}(\bar{x}, \bar{u})>$ 0 , and $(\bar{u}, \bar{\lambda}, \bar{\mu}, \bar{t}) \in W_{2}$, the above inequalities yield

$$
\begin{aligned}
& F\left(\bar{x}, \bar{u} ; \eta^{T}(\bar{x}, \bar{u})\left(\sum_{i=1}^{k} \bar{\lambda}_{i} \nabla f_{i}(\bar{u})+\sum_{j=1}^{m} \bar{\mu}_{j} \nabla g_{j}(\bar{u})\right) P\right) \\
& <-\sum_{i=1}^{k} \frac{\bar{\lambda}_{i} \rho_{i}}{\alpha_{i}(\bar{x}, \bar{u})} d^{2}(\bar{x}, \bar{u}), \\
& F\left(\bar{x}, \bar{u} ; \eta^{T}(\bar{x}, \bar{u})\left(\sum_{j=1}^{m} \bar{\mu}_{j} \nabla g_{j}(\bar{u})\right) P\right) \\
& \quad \leqq-\sum_{j=1}^{m} \frac{\bar{\mu}_{j} \tau_{j}}{\beta_{j}(\bar{x}, \bar{u})} d^{2}(\bar{x}, \bar{u}) .
\end{aligned}
$$
have

Adding both sides of (121) with the sublinearity of $F$, we

$$
\begin{gathered}
F\left(\bar{x}, \bar{u} ; \eta^{T}(\bar{x}, \bar{u})\left(\sum_{i=1}^{k} \bar{\lambda}_{i} \nabla f_{i}(\bar{u})+\sum_{j=1}^{m} \bar{t}_{j} \nabla g_{j}(\bar{u})\right) P\right) \\
<-\left(\sum_{i=1}^{k} \frac{\bar{\lambda}_{i} \rho_{i}}{\alpha_{i}(\bar{x}, \bar{u})}+\sum_{j=1}^{m} \frac{\bar{\mu}_{j} \tau_{j}}{\beta_{j}(\bar{x}, \bar{u})}\right) d^{2}(\bar{x}, \bar{u}), \\
\left(\bar{t}_{j}=2 \bar{\mu}_{j}, j \in M\right) .
\end{gathered}
$$

Using the assumption $\sum_{i=1}^{k}\left(\bar{\lambda}_{i} \rho_{i} / \alpha_{i}(\bar{x}, \bar{u})\right)+\sum_{j=1}^{m}\left(\bar{\mu}_{j} \tau_{j} /\right.$ $\left.\beta_{j}(\bar{x}, \bar{u})\right) \geqq 0$, inequality (122) implies

$$
F\left(\bar{x}, \bar{u} ; \eta^{T}(\bar{x}, \bar{u})\left(\sum_{i=1}^{k} \bar{\lambda}_{i} \nabla f_{i}(\bar{u})+\sum_{j=1}^{m} \bar{t}_{j} \nabla g_{j}(\bar{u})\right) P\right)
$$

$<0$.

By using the constraint condition of (MDII) and $F(\bar{x}, \bar{u} ; 0)=0$, we obtain

$$
\begin{aligned}
& F\left(\bar{x}, \bar{u} ; \eta^{T}(\bar{x}, \bar{u})\left(\sum_{i=1}^{k} \bar{\lambda}_{i} \nabla f_{i}(\bar{u})+\sum_{j=1}^{m} \bar{t}_{j} \nabla g_{j}(\bar{u})\right) P\right) \\
& \quad=0,
\end{aligned}
$$

which is a contradiction to (123). Then $\bar{x}=\bar{u}$.

The proof of part (b) is similar to the proof of part (a).

\section{Discussion and Conclusion}

In this paper, we study the multiobjective programming problems and two kinds of dual models. Then the sufficient optimality conditions, weak dual, strong dual, and strict converse dual, results are obtained and proved under a class of new generalized invex functions assumptions for the multiobjective programming.

\section{Competing Interests}

The authors declare that they have no competing interests.

\section{Acknowledgments}

This work is supported by National Natural Science Foundation of China under Grants no. 71103143 and no. 71473194; Science and Technology Foundation of Shaanxi Province of China under Grant no. 2013XJXX-40; Scientific Research Program Funded by Shaanxi Provincial Education Department (Program no. 15JK1456).

\section{References}

[1] D. S. Kim and K. D. Bae, "Optimality conditions and duality for a class of nondifferentiable multiobjective programming problems," Taiwanese Journal of Mathematics, vol. 13, no. 2, pp. 789-804, 2009.

[2] A. Jayswal, "On sufficiency and duality in multiobjective programming problem under generalized -type I univexity," Journal of Global Optimization, vol. 46, no. 2, pp. 207-216, 2010.

[3] X. Y. Gao, "Optimality and duality for minimax fractional semiinfinite programming," Journal of Networks, vol. 8, no. 3, pp. 739-746, 2013.

[4] K. D. Bae, Y. M. Kang, and D. S. Kim, "Efficiency and generalized convex duality for nondifferentiable multiobjective programs," Journal of Inequalities and Applications, vol. 2010, Article ID 930457, 2010.

[5] D. S. Kim and H. J. Lee, "Optimality conditions and duality in nonsmooth multiobjective programs," Journal of Inequalities and Applications, vol. 2010, Article ID 939537, 13 pages, 2010.

[6] D. B. Ojha, "Second-order duality for nondifferentiable multiobjective programming involving $(\varphi, \rho)$-univexity," Research Journal of Applied Sciences, Engineering and Technology, vol. 2, no. 6, pp. 574-579, 2010.

[7] X. Y. Gao, "Sufficiency in multiobjective programming under second-order B-(p,r)-V-type I functions," Journal of Interdisciplinary Mathematics, vol. 17, no. 4, pp. 385-402, 2014.

[8] A. Jayswal and A. K. Prasad, "Second order symmetric duality in nondifferentiable multiobjective fractional programming with cone convex functions," Journal of Applied Mathematics and Computing, vol. 45, no. 1-2, pp. 15-33, 2014.

[9] X. Y. Gao, "Second order duality in multiobjective programming with generalized convexity," International Journal of Grid and Distributed Computing, vol. 7, no. 5, pp. 159-170, 2014.

[10] T. Antczak, "Optimality and duality for nonsmooth multiobjective programming problems with V-r-invexity," Journal of Global Optimization, vol. 45, no. 2, pp. 319-334, 2009.

[11] T. Antczak, "On G-invex multiobjective programming. Part II. Duality," Journal of Global Optimization, vol. 43, no. 1, pp. 111140, 2009.

[12] T. Antczak, "Optimality conditions and duality for nondifferentiable multiobjective programming problems involving d-r-type I functions," Journal of Computational and Applied Mathematics, vol. 225, no. 1, pp. 236-250, 2009.

[13] C. Nahak and R. N. Mohapatra, "Nonsmooth $\rho-\eta, \theta$-invexity in multiobjective programming problems," Optimization Letters, vol. 6, no. 2, pp. 253-260, 2012.

[14] S. K. Padhan and C. Nahak, "Higher-order symmetric duality with higher-order generalized invexity," Journal of Applied Mathematics and Computing, vol. 48, no. 1-2, pp. 407-420, 2015.

[15] T. Antczak, "Proper efficiency conditions and duality results for nonsmooth vector optimization in Banach spaces under $\phi, \rho$ invexity," Nonlinear Analysis, vol. 75, no. 6, pp. 3107-3121, 2012. 
[16] T. Antczak and A. Stasiak, " $\phi, \rho$-invexity in nonsmooth optimization," Numerical Functional Analysis \& Optimization, vol. 32, pp. 1-25, 2010.

[17] C. L. Yan and B. C. Feng, "Sufficiency and duality for nonsmooth multiobjective programming problems involving generalized $\emptyset, \rho$-V-type I functions," Journal of Mathematical Modelling and Algorithms in Operations Research, vol. 14, no. 2, pp. 159-172, 2015. 


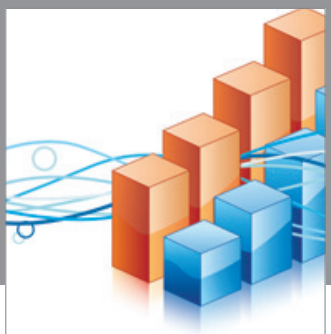

Advances in

Operations Research

vatem alat4

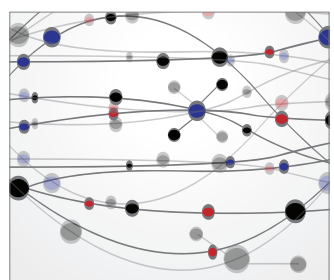

\section{The Scientific} World Journal
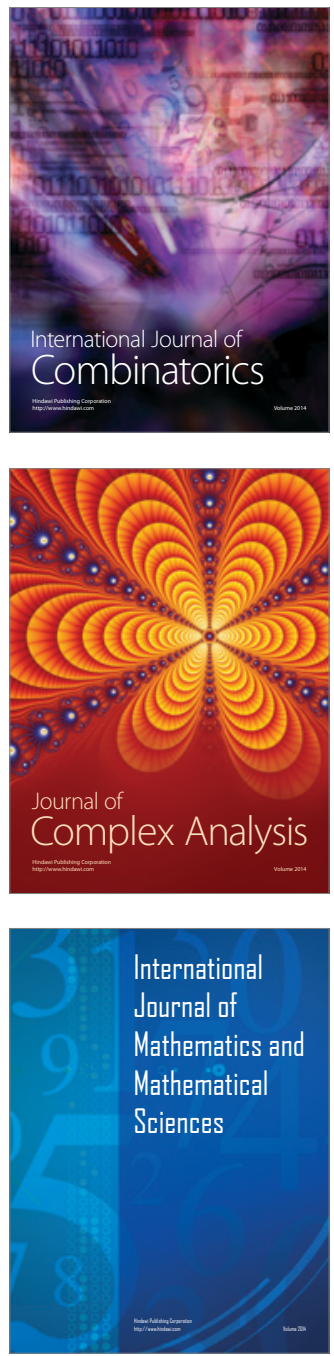
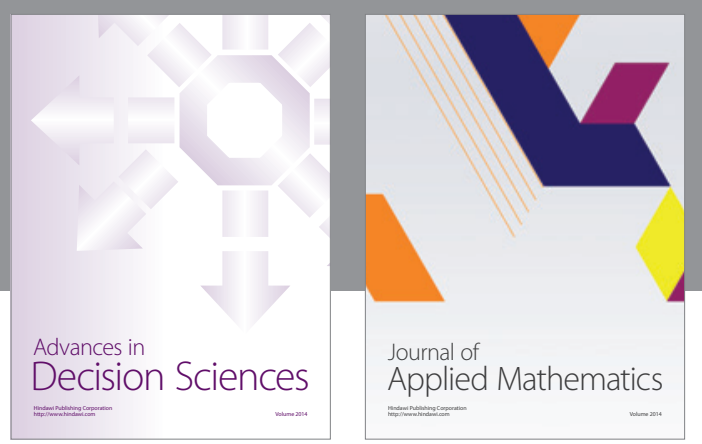

Algebra

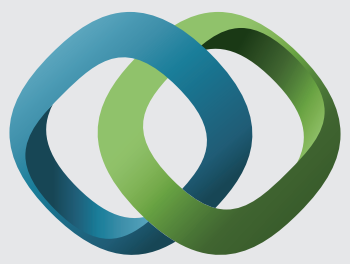

\section{Hindawi}

Submit your manuscripts at

http://www.hindawi.com
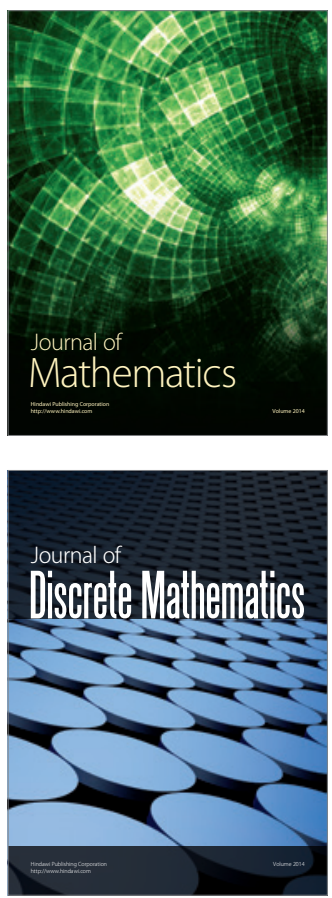

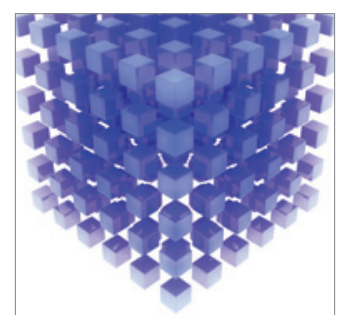

Mathematical Problems in Engineering
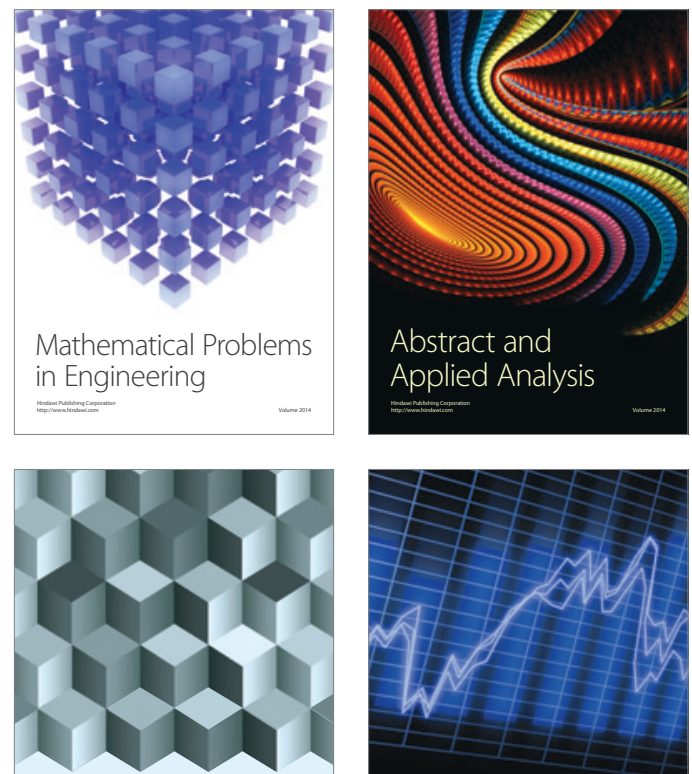

Journal of

Function Spaces

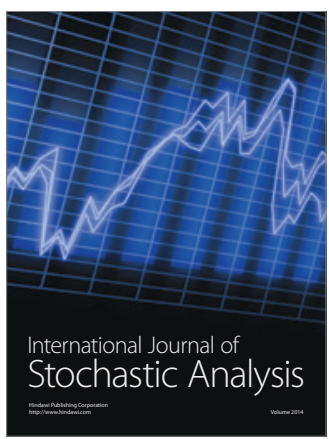

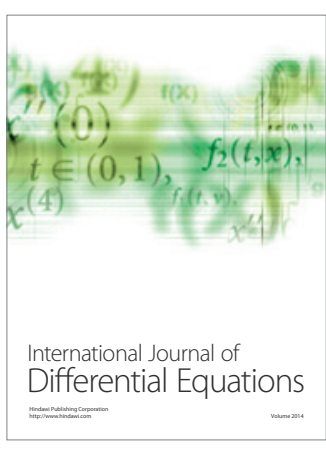
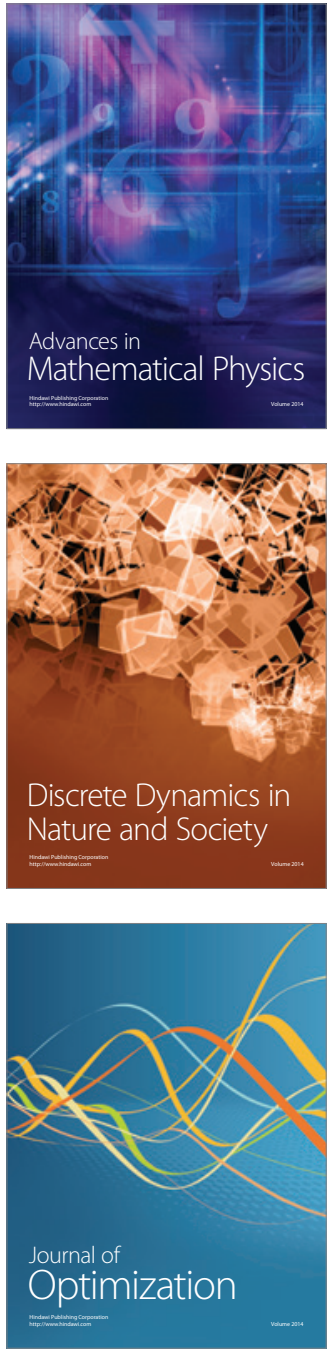